\title{
The Necessity of General Collective Intelligence Driven Processes in Achieving Pervasive Manufacturing
}

\author{
Andy E. Williams, Nobeah Foundation, Nairobi, Kenya
}

\begin{abstract}
General Collective Intelligence has been defined as a system that orchestrates groups to cooperate as a single collective intelligence that greatly increases the group's general problem-solving ability. This increase in group problem-solving ability applies to any group problem. It applies to manufacturing, where GCI has the potential to facilitate decentralized processes not possible otherwise. It applies to design, where GCI has the potential to reliably enable groups to create designs far too complex otherwise. And it applies to cooperation in general, where GCI has the potential to enable cooperation to be reliably scaled, so where the value of that cooperation is positive and can therefore subsidize the cooperation itself, that value might be increased to the point that it can reliably create powerful competitive advantage for groups of local businesses that cooperate to supply local demand through pervasive manufacturing. This paper explores why for these and other reasons, GCI is a necessary component to achieving pervasive use of pervasive manufacturing.
\end{abstract}

\section{Keywords}

General Collective Intelligence, pervasive manufacturing, future of work, automation

\section{Introduction}

Where manufacturing labor is a uniform commodity available everywhere, gaining competitive advantage through increasing centralization, and through the economies of scale allowed by that centralization, can potentially drive manufacturing to the lowest labor cost-centers globally. And where other factors, like the local availability of expertise capable of maintaining it can be eliminated, automation equipment by definition converts manufacturing labor into a purchasable commodity that can be driven to centralize in this way. New technologies with the capacity to customize manufacturing to the individual consumer might also be driven to global centers because of the same efficiencies. Smart manufacturing technologies that introduce opportunities for competitive advantage through increased data capture and increased process control [1] - [5] are among the list of such innovations. While the motivation might be that "anyone can manufacture anywhere", the reality might be different. For example, if industrial-grade additive manufacturing equipment evolves the capacity to manufacture ten thousand parts without significant maintenance costs, it might reliably out-compete consumer grade equipment with the capacity to manufacture ten. However, such customized manufacturing techniques also introduce the possibility of new synergies with the potential to drive massive decentralization [6], [7] so that pervasive manufacturing becomes commercially viable [8], [9], and therefore might become pervasive locally.

To explore where and how this might be the case requires defining a model of the problem to be solved. A business consists of a set of design, manufacturing, and other processes. Physical processes such as manufacturing have material inputs and outputs and are executed manually by people or in an automated way by machinery. Virtual processes such as design have informational inputs and outputs but also might be executed manually by people or in an automated way by computing hardware and software.

There are patterns of solutions to the problem of determining business processes in which pervasive manufacturing can reliably attain a competitive advantage that makes it viable. By determining the 
constraints that must be satisfied in order for these solutions to valid, these solutions can be applied. In this sense, achieving pervasive manufacturing is a kind of boundary value problem.
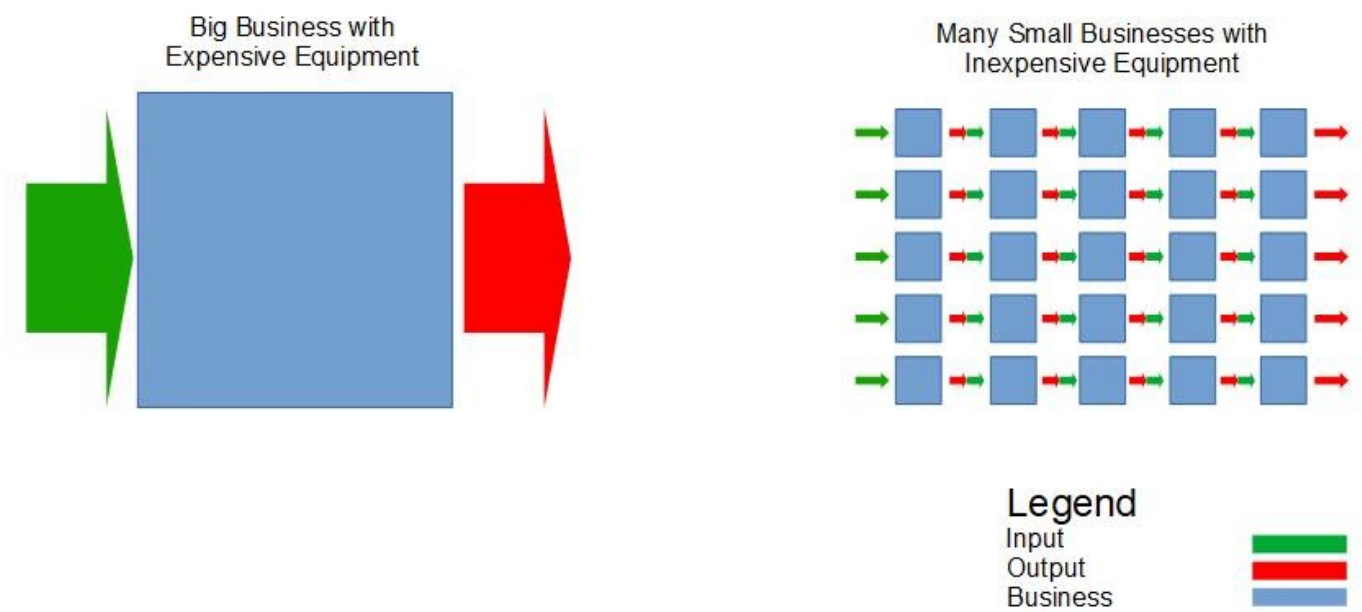

Figure 1: Achieving pervasive manufacturing is a boundary value problem in which the level of decentralized cooperation resulting in a compelling competitive advantage in a given input, output, equipment, or process is the boundary value of cooperation, and solutions must be sought which meet that constraint.

One of these synergies revolves around materials. Customized processes can accommodate locally produced materials. Local consumption of locally produced materials, and local recycling of those materials, creates the potential to cooperate with those producers, recyclers, and others in the value chain. Where cooperation can increase the demand for sustainable access to local materials, and hence increase the value of that access, increasing the capacity for cooperation can reliably increase value.

Another one of these synergies revolves around transportation. Locally produced goods requiring only local transportation can provide faster access to products and services with lower transportation costs. Local transportation, creates the potential to cooperate with local transporters. Where cooperation can add value to local transport, while also increasing the demand for local transport and capacity to provide it, increasing the capacity for cooperation can again reliably increase value.

Yet another one of these synergies revolves around information. There might be a wealth of information available locally that might be used to increase efficiency. While information can be portable, particularly if it is defined semantically, access to information is not generally portable. Furthermore, making all information and reasoning (decision-systems etc.) available to any decentralized process shared by a group of businesses might not be reliably achievable through centralization, which all current group processes without GCI are suggested by the theory of GCI to be driven by. Because according to that theory, decision-making in any group without GCI must tend to become aligned with the interests of the most powerful subset of decision-makers, and at the same time must drive an ever-decreasing subset of decision-makers to become more powerful. Such centralized decision-making can't reliably decentralize access to reasoning processes and other information simply because centralized decision-makers don't reliably see doing so as being in their interests. But in the case of information, where collectively intelligent cooperation with the capacity to maximize group 
outcomes can increase access to information and hence increase the value of that access, increasing the capacity for cooperation through GCI can reliably increase value.

One approach to solving these boundary value problems efficiently enough for the exercise to be reliably feasible, is to define all these problems as the same general problem so they can be solved by the same general problem-solving process with the same general solution. Human-Centric Functional Modeling (HCFM) is an approach that models all static conceptual entities (entities that don't change state) and all dynamic conceptual entities (systems that change state) using a minimally reducible set of functions that maximize generalizability [13] of conceptual models. Using this approach, chains of cooperation involving materials, transportation, information, or other exchanges can be defined as classes of solutions for achieving sufficient local competitive advantage to ensure demand, and those classes generalized for reuse in orchestrating cooperation in other industries [14].

Currently, human beings are the source of the general problem solving ability driving any design or manufacturing process. A recently developed Functional Modeling Framework (FMF) represents the human organism as a problem-solving system consisting of a hierarchy of adaptive problem solving domains. One of these is the domain of adaptation through cognition. Since this is a functional model independent of implementation, it has been used to define a model for implementation in an artificial system (an Artificial General Intelligence or AGI), and it has been used to define a model for implementation in software with the capacity to organize groups into a single collective intelligence (a General Collective Intelligence or GCI) with vastly greater ability to solve group problems.

Another one of these domains is the domain of adaptation through cooperation. Again, since this is a functional model independent of implementation, this model has been applied to represent the cooperation within groups of cells in an individual human, the cooperation in groups of functional components in an AGI, and the cooperation in groups of individuals in a GCI.

Increasing the capacity for groups to reliably converge on selecting whatever product, service, or process that maximizes any group outcome like competitive advantage for the group, requires the capacity to remove the barriers to selecting solutions regardless of whether they require expertise in disciplines unfamiliar to part of the group, whether that selection requires consideration of many more options than any individual in the group has the capacity to evaluate, or whether it involves overcoming any other barriers to scaling cooperation. GCI leverages processes in the domain of cooperation to overcome the barriers to the cooperative execution of processes in the domain of collective cognition. The functions through which GCI overcomes the barriers to group cooperation and group cognition are provided in table 1 .

\section{Component of Model Description}

Functional modeling (problems and solutions) Functional decomposition

Functional domain bridging

Functional fitness

Functional stability
Components are modeled only by function to remove prejudice for or against any given implementation.

Functional components are decomposed into their most basic functional building blocks for reuse.

Different domains in which different functions are more fit in achieving the same purpose are identified. These domains are bridged by using a set of weights which identify the best function in each domain.

Every functional component is assigned some projected and actual fitness in achieving its function.

For functional components to persist they must display some degree of stability in fitness to function. 
For functional components to persist in a changing environment they must have the ability to adapt their function. One of the most important domains of adaptation is cooperation. Functional components must interact according to the principles of decentralized cooperation in order to maximize outcomes for each. Functions must be prioritized according to the principles of centralized cooperation in order to maximize outcomes globally.

Table 1: Components of a HCFM compliant model.

In design, a recent paper has explored why manufacturing or design to achieve collective impacts such as sustainability might require removing the barriers to such collaboration. And therefore, if GCI is necessary to remove the barriers to scaling cooperation, GCI might be necessary in order to reliably achieve sustainability of any product or service [15].

\section{GCI as Necessary Infrastructure for Optimizing the Balance of Centralization and Decentralization of Cooperation}

General problem solving ability requires the ability to adapt to solve different problems. For this reason, where a collective intelligence might use the intelligence of crowds to increase the group's capacity to select the optimal solution to a specific problem or range of problems, a general collective intelligence must also have the capacity to select the optimal problem to solve. One of the most important problems is achieving the pervasive use of GCI, because no matter how good a problemsolving system is in optimizing collective outcomes, it is of no use if it can simply be ignored because it doesn't align with the interests of whatever individual has decision-making authority. From the functional modeling perspective organisms are systems. In designing these systems nature determines which functions the system must have, which components to split this functionality into, and which functionality to put into each functional component. And through this determination nature optimizes the collective response of the cells and other components comprising an organism. In order to ensure that whatever specific components it designs to perform a given function in order to optimize this collective response isn't simply ignored, as in the case with a human ignoring any particular group decision-making system, nature must in other words identify the optimal way for components in systems to cooperate.

Centralization of the cooperation used to make decisions is required to prioritize one participant in cases where the function of one participant has a higher impact on fitness in achieving collective outcomes. Decentralization of the cooperation used to make decisions is required for each participant in the collective to maximize fitness in sustaining any collective outcome. From the perspective of nature, neither centralization nor decentralization are better. The targeted goal is adaptation to improve fitness. However, the theory of GCI suggests that without all of the many layers of decentralization required by an adaptive problem-solving system, problem-solving will tend to become aligned with centralized interests that will circumvent that decentralization. Therefore lack of GCI is a hidden bug preventing all group processes from being collectively optimized with decentralization wherever decentralization is neede. GCI is infrastructure that provides this capacity to optimize cooperation.

\section{Functional Modeling of Products and Services for GCI}

In order for products or services to be accessible to be manipulated by any group process, they must be modeled so they can be consumed ("understood") in a common way by each group process. HumanCentric Functional Modeling (HCFM) defines entities in terms of their human-observable functions rather than through any belief system that might introduce cognitive biases that are not shared, or through any theoretical model that might introduce the necessity of assumptions that are not shared by 
every other theoretical model. This human-centric functional model is achieved through an exercise in "functional decomposition", in which the most universally perceived functions are determined, and a minimal set of these functions capable of being used to compose all other functions is defined so that any model is maximally generalizable.

In addition to defining functions of products and services, products and services are conceptual entities with meaning defined by their relationship to other entities. Since those relationships are defined as reasoning processes that input one set of concepts (the subject of the relationship) and that output another (the object of the relationship), those processes are represented using the common methodology provided in the framework.

\section{Functional Modeling of Design, Manufacturing, and Other Processes Across the Entire Product or Service Life-cycle for GCI}

In order for any group process to be accessible to be manipulated by a GCI, they must be modeled in a common way so they form a general class of problem that the GCI can be used to solve. In the Functional Modeling Framework (FMF) this GCI model is based on, all processes, whether physical or virtual, are modeled in the same way, having inputs, outputs, and other entities that define the context

of execution. Being conceptual representations of these entities, these representations are also defined according to function, as well as being defined by their relationship to other entities. Since those relationships are also defined as reasoning processes that input one set of concepts (the subject of the relationship) and that output another (the object of the relationship), they are also modeled functionally with the same inputs, outputs, and context.

\section{Defining GCI Platforms to Increase Capacity to Orchestrate Cooperation}

Once functional models of products and services have been defined, and once functional models of design, manufacturing, and other life-cycle processes have been defined in order for groups to achieve competitive advantage through collectively intelligent cooperation to execute those processes in relation to those products and services, then implementing those processes requires automating the orchestration of that collectively intelligent cooperation with GCI.

The motivation to do so from the point of view of this paper is not only that certain entire classes of problems might not be reliably definable or solvable without GCI, but in addition that these classes of problems might include pervasive manufacturing and design for pervasive manufacturing, design for sustainability, or design for other purposes. While GCI might be considerable infrastructure, any particular GCI platform might only need to implement a subset of it. For example, the design of the Social Impact Marketplace platform in a recently proposed collective intelligence based agricultural livelihoods program implements only value chain cooperation.

By splitting the GCI functionality over such a number of projects and by implementing such projects in an intelligent sequence, it may be possible to implement it in phases that reduce the cost per project to the point that implementation becomes reliably achievable. A proposed Collective Intelligence based Program to Accelerate Achievement of the Sustainable Development Goals (CIPAA-SDGs) aims to do just that. Where phase I relies only on the Social Impact Marketplace platform, phase II relies on GCI platforms in healthcare, renewable energy, education and other areas. Phase III defines a Collaborative Design platform in order to facilitate a project aiming to leverage massive design collaboration with the capacity to accommodate millions of participants. This project, called "Design for Change Nairobi" aims to use this massive collaboration to lower the cost of designing modular smart, sustainable housing solutions, that incorporate collectively intelligent cooperation with renewable energy, healthcare, or other solutions, to reliably increase income and decrease costs for people in the slums of 
Nairobi by a sufficient amount per month to move those people out of poverty. The principles required to implement collectively intelligent design have been explored elsewhere [15].

In addition, because GCI removes the barriers to scaling cooperation, where the value of that cooperation is positive, that value can then be increased to the point that this cooperation is reliably self-funding. Using this self-funding property, the first three parts of this ten phase program aim to achieve the capacity to reliably drive $\$ 10$ billion USD of private funding towards these goals.

\section{Decentralization and Ownership}

The pervasive capacity for decentralization is required to achieve GCI, and GCI is required to achieve pervasive manufacturing, then an important question is determining the implications of this decentralization on which company structures might be consistent with a GCI. Part of this answer is that any company structure is consistent with GCI because any company can implement any part of GCI that suits it, and can take part in any GCI driven process that suits it, even where other of these processes might implement additional GCI functionality. Another part of this answer is that having the capacity to adapt not only through centralization, but also through decentralization where appropriate, means having complete ownership over decision-making. While it might be possible to develop a platform that orchestrates far more effective cooperation between any one of the two hundred thousand employees of one multi-national company, and any one of the eight hundred thousand employees of another multi-national company, because any decisions involving ownership will be outside of their hands, entire classes of group processes can't be executed, and entire classes of group outcomes are inaccessible.

As one example, in the case of automation, where a centrally owned company of thirty employees who each perform one of thirty tasks, if the owner of that company finds an opportunity for automation, that automation must lead to job loss wherever it consolidates tasks. On the other hand, in a decentralized cooperation of thirty individuals that is bound together by a GCI, if new technology automates a task performed by a subset of individuals, that subset has incentive to band together to purchase that technology and use it where it can increase their own productivity and profitability. The impact of automation is reversed from being a cause of job loss and therefore decrease in collective well-being, to being a source of increase in collective well-being.

The reason is that wherever an impact can be reliably scaled by scaling cooperation, and wherever cooperation can be reliably scaled, then the probability of achieving that impact, and the magnitude of that impact can be increased to the point at which it can reliably be incentivized and reliably occurs. GCI is designed to address all the barriers to scaling cooperation to enable collective impact to be scaled in this way. As an example, if a laptop manufacturing business employs one individual to operate a machine that makes the keys in the keyboard, one individual to operate a machine that makes the case, and one person to assemble them, all three roles might be replaced by a single piece of additive manufacturing (3D printing) equipment. In a GCI based process of cooperation, if it was

optimal for collective well-being to do so, those individuals would be incentivized to band together and purchase that equipment and the skills to operate it, as opposed to the others in the company being incentivized to band together to buy that equipment and eliminate that income opportunity. For this reason, the processes of cooperation in such a GCI based manufacturing business can always be designed so they converge on having this positive collective impact.

\section{Conclusion}

Achieving pervasive decentralized manufacturing, or decentralized massively collaborative design are group outcomes. As discussed in this paper, removing current barriers to achieving any such group 
outcome requires GCI. In turn, the processes and technologies involved in pervasive decentralized design and manufacturing also specifically enable some group outcomes not achievable otherwise, including decentralization of ownership of manufacturing and design companies. It it is true that this decentralization reverses the effect of automation from being a cause of job loss to being a source of collective well-being, this in turn is critical to the future of work. If in addition sustainable and green growth is not reliably achievable without GCI, this is critical to the future of workers.

\section{References}

[1] Shan Ren, Yingfeng Zhang, Yang Liu, Tomohiko Sakao, Donald Huisingh, Cecilia M.V.B. Almeida, A comprehensive review of big data analytics throughout product lifecycle to support sustainable smart manufacturing: A framework, challenges and future research directions, Journal of Cleaner Production, Volume 210, 2019, Pages 1343-1365, ISSN 0959-6526, https://doi.org/10.1016/j.jclepro.2018.11.025.

[2] Yao, X., Zhou, J., Lin, Y. et al. Smart manufacturing based on cyber-physical systems and beyond. J Intell Manuf 30, 2805-2817 (2019). https://doi.org/10.1007/s10845-017-1384-5

[3] Nangia, Shikhil and Makkar, Sandhya and Hassan, Rohail, IoT based Predictive Maintenance in Manufacturing Sector (March 29, 2020). Available at SSRN: https://ssrn.com/abstract=3563559 or http://dx.doi.org/10.2139/ssrn.3563559

[4] Morteza Ghobakhloo, Ng Tan Ching, Adoption of digital technologies of smart manufacturing in SMEs, Journal of Industrial Information Integration, Volume 16, 2019, 100107, ISSN 2452-414X, https://doi.org/10.1016/j.jii.2019.100107.

[5] Andrew Kusiak, Fundamentals of smart manufacturing: A multi-thread perspective, Annual Reviews in Control, Volume 47, 2019, Pages 214-220, ISSN 1367-5788, https://doi.org/10.1016/j.arcontrol.2019.02.001.

[6] X.L. Liu, W.M. Wang, Hanyang Guo, Ali Vatankhah Barenji, Zhi Li, George Q. Huang, Industrial blockchain based framework for product lifecycle management in industry 4.0, Robotics and Computer-Integrated Manufacturing, Volume 63, 2020, 101897, ISSN 0736-5845, https://doi.org/10.1016/j.rcim.2019.101897.

[7] Bogoviz, A.V., Lobova, S.V., Alekseev, A.N., Prokofiev, V.N. and Gimelshtein, I.V. (2019), "Managing the modernization of regional markets of educational services in the conditions of formation of Industry 4.0", On the Horizon, Vol. 27 No. 3/4, pp. 187-192. https://doi.org/10.1108/OTH$\underline{07-2019-0032}$

[8] Holzmann, P., Breitenecker, R.J. and Schwarz, E.J. (2019), "Business model patterns for 3D printer manufacturers", Journal of Manufacturing Technology Management, Vol. ahead-of-print No. ahead-ofprint. https://doi.org/10.1108/JMTM-09-2018-0313

[9] Jyrki Savolainen, Mikael Collan, How Additive Manufacturing Technology Changes Business Models? - Review of Literature, Additive Manufacturing, Volume 32, 2020, 101070, ISSN 2214-8604, https://doi.org/10.1016/j.addma.2020.101070.

[10] Andy E. Williams, A Model for Artificial General Intelligence, in print, Proceedings of the $13^{\text {th }}$ Conference on Artificial General Intelligence (AGI 2020)

[11] Williams, Andy E. “A Model for General Collective Intelligence.” AfricArXiv, 30 Apr. 2020. Web. [12] The Relationship Between Collective Intelligence and One Model of General Collective Intelligence, Andy E. Williams, Computational Collective Intelligence, 11th International Conference, ICCCI 2019, Hendaye, France, September 4-6, 2019, Proceedings, Part II, Pages 589-600 [13] Williams, Andy E. "Use of Human-centric Functional Modeling to Maximize Convergence in Integrative Research.” AfricArXiv, 15 May 2020. Web.

[14] Williams, Andy E. "The Collective Intelligence Based Program to Accelerate Achievement of the Sustainable Development Goals as a Case Study for Collectively Intelligent Program Design."

SocArXiv, 30 Apr. 2020. Web. 
[15] Andy E. Williams, General Collective Intelligence as the Emerging Paradigm in Human-Centric Design for Sustainability, in print, Proceedings of the $26^{\text {th }}$ International Sustainable Development Research Society Conference (ISDRS 2020) 